\title{
An Insight into the Practice of Iron Therapy: Contribution to the On-going Debate with Special Reference to Low- and Middle-income Countries
}

\section{Dr Randa Alsadig Almahdi ${ }^{1}$ and Prof. Dr. Sami A. Khalid ${ }^{2}$}

${ }^{1}$ Assistant Professor Vice dean, Faculty of Pharmacy, University of Science \& Technology Mail address: Faculty of Pharmacy, University of Science and Technology, Omdurman, P O Box 477

${ }^{2}$ Dean, Faculty of Pharmacy, University of science \& Technology Professor of Phytochemistry Faculty of Pharmacy, University of Khartoum

ORCID:

Randa Alsadig Almahdi: http://orcid.org/0000-0002-4319-8112

Corresponding Author: Randa Alsadig Almahdi; email: randaalsiddig2018@outlook.com randa.almahdiust@ust.edu.sd

Received 17 January 2021

Accepted 19 March 2021

Published 31 March 2021

Production and Hosting by Knowledge E

(c) Dr Randa Alsadig Almahdi and Prof. Dr. Sami A.

Khalid. This article is

distributed under the terms of the Creative Commons

Attribution License, which permits unrestricted use and redistribution provided that the original author and source are credited.

Editor-in-Chief:

Prof. Mohammad A. M. Ibnouf

\section{Abstract}

Background: Iron deficiency anemia is a public health problem of a sizable proportion in developing countries. Recently, emerging biochemical knowledge coupled with the discovery of Hepcidin have greatly advanced our understanding of iron metabolism and offered a better insight into its associated pathophysiology. This knowledge should be applied to iron-deficiency anemia therapy to avoid subsequent sequelae of tissue damage associated with reactive oxygen radicals that are catalyzed by iron, because current practices do not include these advances in the treatment guidelines. In the light of recent progress, the existing iron therapy program in many healthcare settings is controversial. This necessitates adjusting the magnitude of iron dose with respect to the very limited iron bioavailability, as gauged by Hepcidin. The current study was therefore aimed to incorporate newly emerging biochemical knowledge into the current iron-deficiency anemia treatment practice

Methods: Literature relevant to iron-deficiency studies published in English between 1964 and 2020 and available online was covered.

Conclusion: Evidently, the existing iron-therapy schedule is both inefficient and toxic. The intricate metabolism of iron should be translated into a more rational iron intervention program with special bias towards low- and middle-income countries requiring a more individualized approach.

Keywords: iron deficiency anemia, hepcidin, oxidative stress, iron therapy

\section{G OPEN ACCESS}

\section{Introduction}

Anemia is a worldwide health problem affecting one-third of the global population, and iron-deficiency anemia (IDA) accounts for half of them [1, 2]. IDA affects populations of 
both developed and developing countries [3, 4]. The developing countries, categorized by the World Bank as low- and middle-income countries (LMICs), present the highest incidence as well as more severe anemia, especially in specific populations [1,5]. The LMICs lack efficient resource allocation; hence they form a large proportion of the world's population with the greatest disease burden [6]. ${ }^{6}$

Iron deficiency in LMICs is the most common micronutrient malnutrition. Its prevalence is high due to poverty that leads to lack of nutritious food, inadequate food intake, inadequate healthcare, diseases, infections, and blood loss [7]. However, in the developed countries, IDA is not a major health problem. In these countries, it only endangers certain groups like young children, adolescents, pregnant women, elderly, blood donors, vegetarians, endurance athletes, and migrants [8].

\section{Materials and Methods}

\subsection{Impact of iron deficiency on the health of vulnerable population}

Iron deficiency occurs when dietary iron intake cannot meet the iron needs over a period of time [9]. The vulnerable population usually includes infants, children with increased growth demands, menstruating girls and women, and pregnant women $[4,10]$. It has been estimated that about half of all pregnant women experience IDA [11], which causes a heavy health burden due to its negative impact on children, coupled with effects on brain development and functioning due to reduced oxygen delivery to tissues [12]. In adults, IDA causes fatigue [13]. Furthermore, severe anemia at hemoglobin levels $<7$ $\mathrm{mg} / \mathrm{dl}$ can result in devastating outcomes, such as maternal mortality, reduced birth weight, and reduced cognitive ability [10].

\subsection{Common causes of iron deficiency in LMICs}

The populations in the LMICs are usually characterized by nutritional deficiencies, low iron-containing food, or problems in absorption of dietary iron due to viral and parasitic infections, for example, malaria (since these pathogenic microbes consume iron as well), and high prevalence of Helicobacter pylori infection [14]. Moreover, IDA may arise secondary to partial gastrectomy, coeliac disease, Crohn's disease, some drugs that reduce iron absorption [15], hemorrhage associated with childbirth, or heavy menstrual blood loss. It can also result secondary to impaired absorption due to high intake of 
phytate that impairs iron absorption or altered nutrient metabolism [12]. Malnutrition causes a wider segment of the population in LMICs to be at risk of IDA.

A recently identified risk factor for IDA is oxidative stress induced by high unbound iron that eventually damages erythrocytes and results in IDA [16, 17].

\subsection{Iron: An integral component for cellular functions}

Iron is a biologically essential component for all living organisms. In humans, it exists as protein-bound, heme compounds, which include hemoglobin containing $25 \%$ of the body iron and myoglobin containing 15\% [18], or non-heme compounds like transferrin and ferritin [19]. Heme enzymes and other iron-containing enzymes are involved in electron transfer and oxidation-reduction processes [20]. The human body obtains iron from three sources: environment (mother to fetus), recycled or mobilized form from iron storage sites (major source), and diet (limited source) [21-25].

The quantity of iron in the body is well-regulated and tightly controlled through a robust network at both cellular and systemic levels [26]. Most of the iron in the body is recycled and reserved within the body, leaving only a small quantity to be acquired exogenously either from diet or iron supplements with limited bioavailability [27].

Iron has always shown to be an intricate element in conflicting mechanisms. Iron can be described as beneficial as a micronutrient, as well as harmful to body tissues as a catalyst in a series of reactions that produce the toxic reactive oxygen species (ROS) $[26,28]$. Therefore, both iron deficiency and overload can have serious health effects [29]. This paradoxical role has always confronted scientists regarding the body's mechanisms to coordinate iron delivery to tissues $[30,31]$

To understand the biochemical mechanisms involved in iron absorption and the various factors associated with these processes, two facts should be considered. Most of the iron in the body is reserved within the body and recycled from different storage sites, including senescent erythrocytes to other tissues. This implies that iron from exogenous sources has very limited bioavailability [27]. This entails that iron trafficking requires effective tissue communication and orchestration between the iron consuming cells, for example, erythrocyte precursors, and the iron storage sites, for example, duodenal enterocytes, hepatocytes, and tissue macrophages [2]. 


\subsection{Absorption of iron from the gut}

Absorption is the most important mechanism in iron haemostasias [7]. Iron haemostasias has been described in the literature as a partially understood process. Therefore, it has been subjected to extensive research for decades to fill the gaps of knowledge about its absorptive mechanisms [2]

Dietary iron is available in two main forms: organic haem iron and non-haem inorganic iron [4, 32]. The non-haem inorganic iron is available mostly in oxidized ferric form besides its availability in its reduced ferrous form [33]. The duodenum and upper jejunum have long been identified as the absorptive sites for iron [22]. Initially, ferrous iron is up-taken in the gut by the enterocytes through the protein carrier DMT-1 [20], while ferric iron is reduced to ferrous before being up-taken or carried by a different pathway (mobilferrin-integrin) [32]. The ferrous complex with DMT-1 is influenced and regulated to a great extent by iron deficiency, whereas the ferric-mobilferrin-integrin complex is not [23].

Iron exits from the enterocytes into the plasma via the export protein ferroportin $[19,27]$, and it is then carried by transferrin to either fuel erythropoiesis in the bone marrow or to be stored in ferritin [27]. Eventually, the quantity of iron that actually reaches plasma is the iron released from the enterocytes as transferrin-bound iron via ferroportin and not that up-taken by the enterocytes from the gut [34]

The level of amount of iron absorbed is maintained by cellular and systemic pathways [35]. At the cellular level of the mucosal block, large quantities of iron are detained by enterocyte ferritin, a natural nano-protein mucosal acceptor. Thus, one ferritin molecule cages 4,500 iron atoms [15, 25], while the excess iron stored in ferritin eventually gets unloaded in the gut when enterocytes get sloughed at the end of their life span [36]. Signaled by the quantities of intracellular iron [23, 27, 32, 37-39], a reduction in brush border enzymes occurs resulting in inhibition of iron absorption for more than 4-5 hr after a single oral iron dose [39]. The systemic protective mechanism against excess iron is controlled by hepcidin. Therefore, we will elaborate on the important discovery of hepcidin as a safety gauge.

Inside the cell, an important fraction of iron known as labile iron pool (LIP) remains bound to low molecular weight chelates, such as citrates, adenosine triphosphate, ascorbic acid, or pyrophosphate. Usually, LIP represents $<5 \%$ of total cellular iron at iron blood level of 50-100 $\mu \mathrm{M}$ [26], however, its quantity may rise substantially by the induction of certain biochemical stimuli, such as iron overload or iron levels that exceed cell haemostasias $[26,30]$ 


\subsection{Gauging iron absorption (inhibition vs enhancement)}

Exogenous iron absorption is largely dictated by the body's iron requirement [23]. Consequently, the absorption of iron from the gut or mobilization from storage sites is accelerated by plasma iron turnover to produce more erythrocytes in response to stimulation by the erythropoietin hormone $[2,23]$. Nevertheless, absorption of iron is influenced by many other factors and substances coexisting in the gut, either by inhibition or enhancement. Enhancers include ascorbic acid, meat, and poultry, while inhibitors are tannins, dairy products, polyphenols, and phytates [40, 41], as well as some trace elements like zinc and copper, which are found in the enzymes involved in iron metabolism $[12,41]$.

\subsection{Damaging role of oxidative stress triggered by free (unbound) iron}

LIP is chelatable transient iron and a critical factor in producing catalytic iron that generates ROS and nitrogen species through the Haber-Weiss reaction [26, 28], which is responsible for initiating the Fenton's reaction [30, 42]. Fenton's reaction is considered as a major mechanism for generating ROS in biological systems [43]. It causes corruption of the lipid membranes, proteins, and DNA [44]. Peroxidation of membrane lipids can damage the membrane structure, decrease the cell deformability, enhance immunoglobulin-G binding, and complement activation [45]. This damage promotes a state of oxidative stress [26, 37], and eventually leads to degradation of erythrocytes that damages tissues and organs $[15,16]$. Oxidative stress can be induced by high intake of iron during iron supplementation with doses that exceed the capacity of endogenous antioxidant proteins (e.g., glutathione), which could lead to the generation of ROS. This could result in tissue damage, specifically of the intestinal mucosa that is vulnerable to oxidative damage [45], inflammation of the colon, and disturbances of the microbiome [46].

Generally, our body is capable of detoxification and neutralization of ROS by the naturally occurring endogenous antioxidants to restore the pro-oxidants-antioxidants balance and prevent the damage of oxidative stress. Moreover, other natural protective mechanisms exist to prevent the oxidative stress related to free unbound iron through hepcidin and chelation of LIP iron [30, 35, 42].

Recently, there has been equivocal evidence about the important role of oxidative stress in the pathophysiology of IDA through increased free cellular iron [47] and the 
systemic and cellular handling of iron haemostasias [27, 39, 48, 49]. This new knowledge causes the co-ingestion of exogenous antioxidants to become a rational adjunctive to iron therapy [50].

\subsection{Strategies to control iron-deficiency anemia}

The persistence of IDA as a worldwide health problem requires a novel and innovative interventional approach by the provision of appropriate and bioavailable forms of iron dosage that avoid iron toxicity. Meanwhile, several constraints exist in the resources of poor health settings in LMICs, including proper diagnosis, prevention, and effective management of IDA. Iron replete is crucial for healthy development of children and the well-being of all people in LMICs. Therefore, it is especially important to develop strategies mainly addressing LMICs to protect such vulnerable populations from the negative impacts of IDA.

Historically, there were different approaches to control IDA, including food fortification and iron salts supplementation [51]. Despite the continuous efforts by various international organizations to come up with appropriate guidelines on iron supplementation, and more than 17 guidelines proposed by the World Health Organization alone [12], none of these guidelines are based on evidence-based clinical study.

Implementation of anemia control programs in LMICs requires careful baseline epidemiologic evaluation to select the appropriate interventions that suit the most affected populations, in line with the currently available scientific evidence to ensure safety and effectiveness [5].

To meet iron requirements in LMICs where iron deficiency is widespread, adequate intervention programs are recommended. However, iron is known to influence the pathogenesis of a number infectious disease (e.g., malaria), which are especially common among the populations of the LMICs [51, 52]. Earlier evidence has already demonstrated the dose-related deleterious effect of iron therapy in malaria endemic regions [53].

In some of the LMICs with a high malaria burden, hepcidin blocks iron absorption from the gut as well as inhibits its mobilization, resulting in prevention of efficient iron uptake. This means that iron supplementation in malaria endemic areas would be less effective [34]. This represents a challenge for policy-makers, and the urgent need to develop efficient therapeutic approaches with minimal infection-related risks. Moreover, it requires individualizing the therapeutic regimen according to iron deficiency, infection risk, and inflammatory status of the individuals [52] 


\subsection{Pathophysiology of iron-deficiency anemia}

Iron deficiency develops gradually in states of negative iron balance in three stages. It typically evolves through storage iron depletion (latent anemia), iron-deficient erythropoiesis, and finally overt iron-deficiency anemia (defined as concomitant iron deficiency plus anemia) [54].

\subsection{Diagnosis of iron-deficiency anemia in LMICs}

The most commonly used indicator of IDA in many poor resource settings like the LMICs is hemoglobin. However, to confirm iron deficiency in patients with low hemoglobin, a low serum ferritin level must be established $[10,55]$. In cases of coexisting infectious disease and/or inflammation, serum ferritin, which is an acute phase reactant, may give false results [56], because its levels increase in infection and/or inflammation [12, 57]. In such situations, a simpler approach that optimally uses serum ferritin in IDA diagnosis is considering values $<15 \mu \mathrm{g} / \mathrm{dl}$ as diagnostic for IDA and those $>100 \mu \mathrm{g} / \mathrm{dl}$ to rule out IDA [58]. Alternatively, other inflammatory markers should be included in the assessment, such as C-reactive protein and alpha-1 glycoprotein [12], considering that transferrin saturation is a very sensitive parameter to evaluate body iron stores [45].

IDA should be classified to determine its severity for appropriately guided therapy. There are a number of cut-off values for hemoglobin to determine severity of IDA, for example, mild IDA at hemoglobin of 10-10.9 $\mathrm{g} / \mathrm{dl}$, moderate IDA at hemoglobin level of 7-9.9 g/dl, and severe IDA at levels $<7 \mathrm{~g} / \mathrm{dl}$ [13].

\subsection{Iron supplementation scheme}

After a confirmed IDA diagnosis, the longstanding trend of treatment is iron supplementation either by oral or intravenous route. The choice is largely dependent on factors such as the patient's age, sex, severity of anemia, and timeframe for acceptable iron correction [59].

The orthodox treatment of IDA to replenish the iron stores fully is oral administration of 100-200 mg elemental iron twice per day [39, 60], for three months [4, 54]. The oral route has always been the first-line choice due to its safety, cost-effectiveness, and convenience [61]. Frequently used oral iron salts, such as ferrous fumarate, ferrous sulphate, and ferrous gluconate, have similar bioavailability and slightly different elemental iron content [60]. 


\subsection{Current debate on iron therapy practice}

The magnitude and spacing of oral iron dose are becoming issues of great concern and controversy due to the inefficiency and toxicity of the iron dose in use [27, 39]. Based on the discovery of hepcidin, Moretti et al. have questioned the need for large daily doses of iron as compared to the much smaller dose of bioavailable iron at $12.5 \%$ of the dose. This has raised safety concerns regarding the potential risk of oxidative stress and involvement of unbound iron in Fenton's reaction [26, 61]. This concern has been addressed and consolidated experimentally for the first time by a good evidencebased clinical trial [39]. It is necessary to remember that pregnant women are among the vulnerable groups for IDA due to the higher risk of potential iron toxicity related to oxidative stress that is associated with the mitochondria-rich placenta [61].

Oral iron salts remain the standard IDA treatment option in current use, associated with dose-dependent toxicity [57], and subsequently have been known to compromise compliance to treatment. Therefore, there is a need for a better and safer treatment options to replenish iron stores in IDA patients.

\subsection{Call for a safer and more effective iron therapy}

The oral route remains the first-line choice in the wider population of patients [46]. Its advantages include cost-effectiveness and ease of monitoring, especially in health settings with limited resources [64]. The challenge for IDA treatment in the poor-resource settings of LMICs are facing many constrains with respect to the diagnosis, prevention, and treatment of IDA. Effective management often requires treatment of the underlying cause prior to iron supplementation, restoration of hemoglobin to normal levels, and treatment of complications if they exist [65].

Many reviews and studies have concluded that there is an urgent and compelling need to revisit the iron therapy guidelines that are currently in use $[65,66]$. Some have proposed recommendations for safer regimens [39, 45, 65]. Recent reviews are in favor of smaller iron doses and intermittent dosing rather than daily doses [67].

With respect to iron supplementation, it is important to bear in mind that certain individuals may be at a higher risk with high iron doses, such as severely ill patients with inflammatory diseases [68], IDA patients with chronic renal diseases [69], and patients with underlying infections or malignancy [70]. In such patients, iron supplementation may be deleterious and/or even harmful [70]. 


\section{Conclusion}

The discovery of hepcidin and its role in IDA should be considered as a turning point in iron therapy and a great advance in our knowledge about IDA. It is of paramount importance to consider this in therapeutic decisions while determining the iron dose, spacing, and duration, to avoid inefficiency of iron bioavailability, for example, twice per week or intermittent dosing. Furthermore, the potential of high iron supplementation to increase free unbound iron and fuel the deleterious effect of catalyst iron in Fenton's reaction is indicative of the need to reduce the iron dose magnitude.

From the aforementioned information, it can be concluded that what matters most with iron therapy is not iron replete, but rather the iron supplementation with an appropriate methodology.

Development of efficient and rational iron therapy program in LMICs should be done through individualized approaches with special concern to the widespread infectionrelated health issues, for example, malaria, tuberculosis, and HIV infections, which have negative interactions with iron therapy.

\section{Acknowledgements}

\section{Ethical considerations}

Not applicable.

\section{Competing interests}

The authors declare that there is no conflict of interest.

\section{Availability of data and material}

The data that support the findings of this study are available from the corresponding author upon request and most are openly available at the provided URL addresses and doi links

\section{Funding}

None. 


\section{References}

[1] World Health Organization and United Nation Children's Fund. (2004). Towards an Integrated Approach for Effective Anemia Control. Focusing on Anemia. Retrieved from: https://www.who.int/nutrition/publications/micronutrients/ WHOandUNICEF_statement_anaemia/en

[2] Lopes, T. S., Lugonskaja, T., Spasic, M. V., et al. (2010). Systems analysis of iron metabolism: the network of iron pools and fluxes. BMC Systems Biology, vol. 4, pp. 112-130. Retrieved from: https://link.springer.com/article/10.1186/1752-0509-4-112

[3] Ramakrishnan, U. and Yip, R. (2002). Experiences and challenges in industrialized countries: control of iron deficiency in industrialized countries. Journal of Nutrition, vol. 132, no. 4, pp. 820-824. Retrieved from: https://pubmed.ncbi.nlm.nih.gov/ $11925488 /$

[4] Umbreit, J. (2005). Iron deficiency: a concise review. American Journal of Hematology, vol. 78, pp. 225-231. Retrieved from: https://pubmed.ncbi.nlm.nih.gov/ 15726599/

[5] Pasricha, S. R., Drakesmith, H., Black, J., et al. (2013). Control of iron deficiency anemia in low and middle income countries. Blood, vol. 121, no. 14, pp. 2607-2616. Retrieved from: https://ashpublications.org/blood/article/121/14/2607/31112/Controlof-iron-deficiency-anemia-in-low-and-middle-income-countries

[6] Kularatna, S., Whitty, J. A., Johnson, N. W., et al. (2013). Health state valuation in low-and middle-income countries: a systematic review of the literature. In: R. M. Scheffler (Ed.). World Scientific Handbook of Global Health Economics and Public Policy (p. 1091-1099). Singapore: World scientific Publishing Co. Pte. Ltd. Retrieved from: https://pubmed.ncbi.nlm.nih.gov/24041360/

[7] Curis, C. (2013). Iron Supplementation in Nutrition Programs: Pathophysiological Basis and Correlations with Health in Developing Countries. Université de Lyon. Retrieved from: http://biologie.ens-lyon.fr/ressources/bibliographies/m1-12-13biosci-reviews-curis-c-1c-m.xml

[8] Marx, J. M. (1997). Iron deficiency in developed countries: prevalence, influence of lifestyle factors and hazards of prevention. European Journal of Clinical Nutrition, vol. 51, pp. 491-494. Retrieved from: https://pubmed.ncbi.nlm.nih.gov/11248872/

[9] Clark, S. F. (2008). Iron deficiency anemia. Nutrition in Clinical Practice, vol. 23, no. 2, pp. 128-141.

[10] Ramakrishnan, U. and Imhoff-Kunsch, B. (2008). Anemia and iron deficiency in developing countries. In: C. J. Lammi-Keefe, S. C. Couch, E. H. Philipson (Eds.), 
Handbook of Nutrition and Pregnancy (pp. 337-354). Humana Press.

[11] International Nutritional Anemia Control Group (INACG), The World Health Organization (WHO), United Nations Children's Fund (UNICEF). (2011). Guidelines for Use of Iron Supplements to Treat and Prevent Iron Deficiency Anemia. Retrieved from: https://www.who.int/nutrition/publications/micronutrients/ anaemia_iron_deficiency/1-57881-020-5/en/

[12] Chaparro, C. M. and Suchdev, P. S. (2019). Anemia epidemiology, pathophysiology, and etiology in low- and middle-income countries. Annals of the New York Academy of Sciences, vol. 1450, no. 1, pp. 15-31. Retrieved from: https://www.ncbi.nlm.nih.gov/ pmc/articles/PMC6697587/

[13] Killip, S., Bennet, J. M., and Chambers, M. D. (2007). Iron deficiency anemia. American Family Physician, vol. 75, no. 5, pp. 671-678. Retrieved from: https://www.aafp.org/ afp/2007/0301/p671.html

[14] Qu, X. H., Huang, X. L., Xiong, P., et al. (2010). Does Helicobacter pylori infection play a role in iron deficiency anemia? A meta-analysis. World Journal of Gastroenterology, vol. 16, no. 7, pp. 886-896. Retrieved from: https://www.ncbi.nlm.nih.gov/pmc/ articles/PMC2825337/

[15] Yiannikourides, A. and Latunde-Dada, G. O. (2019). A short review on iron metabolism and pathophysiology of iron disorders. Medicines, vol. 6, no. 3, p. 85. Retrieved from: https://www.mdpi.com/2305-6320/6/3/85

[16] luchi, Y. (2012). Anemia caused by oxidative stress. In: D. S. Silverberg (Ed.) [Internet]. London: IntechOpen. Retrieved from: https://www.intechopen.com/books/anemia/ anemia-caused-by-oxidative-stress. doi: 10.5772/31404

[17] Mishra, K., Ojha, H., and Chaudhary, N. K. (2012). Estimation of antiradical properties of antioxidants using DPPH assay: a critical review and results. Food Chemistry, vol. 130, no. 4, pp. 1036-1043. Retrieved from: https://www.sciencedirect.com/science/ article/abs/pii/S0308814611011058

[18] Munoz, M., Villar, I., and Garcia-Erce, J. A. (2009). An update on iron physiology. World Journal of Gastroenterology, vol. 15, no. 37, pp. 4617-4626. Retrieved from: https://www.ncbi.nlm.nih.gov/pmc/articles/PMC2754509/

[19] Ma, Y., Yeh, M., Yeh, K., et al. (2006). Iron Imports. V. transport of iron through the intestinal epithelium. American Journal of Physiology-Gastrointestinal and Liver Physiology, vol. 290, no. 3, pp. 417-422. Retrieved from: https://journals.physiology. org/doi/full/10.1152/ajpgi.00489.2005

[20] Abbaspour, N., Hurrell, R., and Kelishadi, R. (2014). Review on iron and its importance for human health. Journal of Research in Medical Sciences, vol. 19, no. 2, pp. 164-174. 
Retrieved from: https://www.ncbi.nlm.nih.gov/pmc/articles/PMC3999603/

[21] Crosby, W. H. (1963). Editorial review: the control of iron balance by the intestinal mucosa. Blood, vol. 22, no. 4, pp. 441-449. Retrieved from: https://ashpublications. org/blood/article/22/4/441/37810/Editorial-Review-The-Control-of-Iron-Balance-by

[22] Wheby, M. S., Jones, L. G., and Crosby, W. H. (1964). Studies on iron absorption. Intestinal regulatory mechanisms. Journal of Clinical Investigation, vol. 43, no. 7, pp. 1433-1442. Retrieved from: https://www.ncbi.nlm.nih.gov/pmc/articles/PMC289619/

[23] Conard, M. E. and Umbreit, J. N. (2000). Iron absorption and transport-an update. American Journal of Hematology, vol. 64, no. 4, pp. 287-298. Retrieved from: https://onlinelibrary.wiley.com/doi/abs/10.1002/1096-8652(200008)64:4\{\%\}3C287:: AID-AJH9\{\%\}3E3.0.CO;2-L

[24] Ganz, T. (2005). Cellular iron: ferroportin is the only way out. Cell Metabolism, vol. 1, no. 3, pp. 155-157. Retrieved from: https://www.sciencedirect.com/science/article/ pii/S1550413105000586?via\{\%\}3Dihub

[25] Cui, Y., Wu, Q., and Zhou, Y. (2009). Iron refractory iron deficiency: new molecular mechanisms. Kidney International, vol. 76, no. 11, pp. 1137-1141. Retrieved from: https: //www.sciencedirect.com/science/article/pii/S0085253815538830

[26] Puntarulo, S. (2005). Iron, oxidative stress and human health. Molecular Aspects of Medicine, vol. 26, no. 4-5, pp. 299-312. https://www.sciencedirect.com/science/ article/abs/pii/S009829970500035X

[27] Ganz, T. (2011). Hepcidin and iron regulation, 10 years later. Blood, vol. 117, no. 17, pp. 4425-4433. Retrieved from: https://www.ncbi.nlm.nih.gov/pmc/articles/ PMC3099567

[28] Udipi, S., Ghugra, P., and Gokhale, C. (2012). Iron, oxidative stress and health. In: C. Gokhale (Ed.), Molecular Mechanisms and Biological Effects (pp. 73-109) [Internet]. India: IntechOpen.

[29] Pra, D., Franke, S. I., Henriques, J. A., et al. (2012). Iron and genome instability: an update. Mutation Research, vol. 733, no. 1-2, pp. 92-99. Retrieved from: https: //www.sciencedirect.com/science/article/pii/S0027510712000413

[30] Kakhlon, O. R. and Cabantchik, Z. L. (2002). The labile iron pool: characterization, measurement and participation in cellular processes. Free Radical Biology and Medicine, vol. 33, no. 8, pp. 1037-1046. Retrieved from: https://www. elsevier.com/search-results?query=30.\{\%\}09KakhlonOR\{\%\}2C\{\%\}20Cabantchik. TheLabilelronPool\{\%\}3A

[31] Hentze, M. W., Muckenthaler, M. U., and Andrews, N. C. (2004). Balancing acts: molecular control of mammalian iron metabolism. Cell, vol. 117, no. 3 , 
pp. 285-297. Retrieved from: https://www.sciencedirect.com/science/article/pii/ S0092867404003435

[32] Benito, P. and Miller, D. (1998). Iron absorption and bioavailability: an updated review. Nutrition Research, vol. 18, no. 3, pp. 581-603. Retrieved from: https: //www.sciencedirect.com/journal/nutrition-research/vol/18/issue/3

[33] Johnson-Wimbley, T. D. and Graham, D. Y. (2011). Diagnosis and management of iron deficiency anemia in $21^{\text {st }}$ century. Therapeutic Advances in Gastroenterology, vol. 4, no. 3, pp. 177-184. Retrieved from: https://journals.sagepub.com/doi/abs/10.1177/ $1756283 \times 11398736$

[34] Spottiswoode, N., Duffy, P. E., and Drakesmith, H. (2014). Iron, anemia and hepcidin in malaria. Frontiers in Pharmacology, vol. 5, pp. 125-136. Retrieved from: https: //www.frontiersin.org/articles/10.3389/fphar.2014.00125/full

[35] Kim, J. and Wessling-Resnick, M. (2012). The role of iron metabolism in lung inflammation and injury. Journal of Allergy \& Therapy, vol. 3, no. 4, p. 004. Retrieved from: https://dash.harvard.edu/handle/1/34652039

[36] Theil, E. C., Chen, H., Miranda, C., et al. (2012). Absorption of iron from ferritin is independent of heme iron and ferrous salts in women and rat intestinal segments. Nutrition Journal, vol. 142, no. 3, pp. 478-483. Retrieved from: https://academic.oup. com/jn/article/142/3/478/4743475

[37] Brown, E. B. (1963). The absorption of iron. American Journal of Clinical Nutrition, vol. 12, no. 3, pp. 205-213. Retrieved from: https://academic.oup.com/ajcn/articleabstract/12/3/205/4823082

[38] Frazer, D. M., Wilkins, S. J., Becker, E. M., et al. (2003). A rapid decrease in the expression of DMT1 and Dcytb but not Ireg1 or Hephaestion explains the mucosal block phenomenon of iron absorption. Gut, vol. 52, no. 3, pp. 340-346. Retrieved from: https://gut.bmj.com/content/52/3/340.short

[39] Moretti, D., Goede, J. S., Zeder, C., et al. Oral iron supplements increase hepcidin and decrease iron absorption from daily or twice daily doses in iron depleted young women. Blood 2015, vol. 126, no. 17, pp. 19811989. Retrieved from: https://ashpublications.org/blood/article/126/17/1981/34441/ Oral-iron-supplements-increase-hepcidin

[40] Minihane, A. M. and Rimbach, G. (2002). Iron absorption and the iron binding and antioxidant properties of phytic acid. International Journal of Food Science \& Technology, vol. 37, no. 7, pp. 741-748. Retrieved from: https://ifst.onlinelibrary. wiley.com/doi/full/10.1046/j.1365-2621.2002.00619.x 
[41] Collings, R., Harvey, L. J., Hooper, L., et al. (2013). Absorption of iron from whole diet: a systematic review. American Journal of Clinical Nutrition, vol. 98, no. 1, pp. 65-81. Retrieved from: https://academic.oup.com/ajcn/article/98/1/65/4578342

[42] Kruszewski, M. (2003). Labile iron pool: the main determinant of cellular response to oxidative stress. Mutation Research - Fundamental and Molecular Mechanisms, vol. 531, no. 1-2, pp. 81-92. Retrieved from: https://www.sciencedirect.com/science/ article/pii/S0027510703001623

[43] Kehrer, J. P. (2000). The Haber-Weiss reaction and mechanism of toxicity. Toxicity, vol. 149, no. 2000, pp. 43-50. Retrieved from: https://www.sciencedirect.com/science/ article/abs/pii/S0300483X00002316

[44] De Bono, D. P. (1994). Free radicals and antioxidants in vascular biology: the role of reaction kinetics environment and substrate turnover. QJM, vol. 87, no. 8, pp. 445-453. Retrieved from: https://academic.oup.com/qjmed/article-abstract/87/ $8 / 445 / 1599126$

[45] Pavord, S., Myers, B., Robinson, S., et al. (2012). UK guidelines on the management of IDA in pregnancy. British Journal of Haematology, vol. 156, no. 5, pp. 588-600. Retrieved from: https://onlinelibrary.wiley.com/doi/full/10.1111/j.1365-2141.2011.09012. $x$

[46] Auerbach, M., Mentzer, W. C., Tirnauer, J. S., et al. (2020). Causes and Diagnosis of Iron Deficiency and Iron Deficiency Anemia in Adults. UpToDate.com. Retrieved from: https://www.uptodate.com/contents/causes-and-diagnosis-of-iron-deficiencyand-iron-deficiency-anemia-in-adults

[47] Aslan, A., Horoz, M., and Celik, H. (2011). Evaluation of oxidative status in iron deficiency anemia through total antioxidant capacity measured using an automated method. Turkish Journal of Hematology, vol. 28, no. 1, pp. 42-46. Retrieved from: https://go.gale.com/ps/anonymous?id=GALE\{\TUltextbar\}A305562773\&sid

[48] Schrier, S. L. (2015). So you know how to treat iron deficiency anemia. Blood, vol. 126, no. 17, p. 1971. Retrieved from: https://ashpublications.org/blood/article/126/17/ 1971/34416/So-you-know-how-to-treat-iron-deficiency-anemia

[49] Liu, J., Sun, B., Yin, H., et al. (2016). Hepcidin: a promising therapeutic target for iron disorders: a systematic Review. Medicine, vol. 95, no. 14, p. e3150. Retrieved from: https://www.ncbi.nlm.nih.gov/pmc/articles/PMC4998755/

[50] Pham-Huy, L. A., He, H., and Pham-Huy, C. (2008). Free radicals, antioxidants in disease and health. International Journal of Biomedical Science, vol. 4, no. 2, pp. 89-96. Retrieved from: https://www.ncbi.nlm.nih.gov/pmc/articles/PMC3614697 
[51] Brittenham, G. M. (2012). Safety of iron fortification and supplementation in malariaendemic areas. Nestle Nutrition Institute Workshop Series, vol. 70, pp. 117-127. Retrieved from: https://europepmc.org/article/med/25762976

[52] Armitage, A. E. and Moretti, D. (2019). The importance of iron status for young children in low and middle income countries: a narrative review. Pharmaceuticals, vol. 12, no. 2, p. e59. Retrieved from: https://www.mdpi.com/1424-8247/12/2/59

[53] Oppenheimer, S. J. (2001). Iron and its relation to immunity and infectious disease. Nutrition Journal, vol. 131, no. 2, pp. S616-S633. Retrieved from: https://pubmed. ncbi.nlm.nih.gov/11160594/

[54] Short, M. W. and Domagalski, J. E. (2013). Iron deficiency anemia: evaluation and treatment. American Family Physician, vol. 87, no. 2, pp. 98-104. Retrieved from: https://www.aafp.org/afp/2013/0115/p98.html

[55] Denic, S. and Agarwal, M. M. (2007). Nutritional iron deficiency: an evolutionary perspective. Nutrition, vol. 23, no. 7-8, pp. 603-614. Retrieved from: https://pascalfrancis.inist.fr/vibad/index.php?action=getRecordDetail\&idt=19903993

[56] Wish, J. B. (2006). Assessing iron status beyond serum ferritin and transferrin saturation. Clinical Journal of the American Society of Nephrology, vol. 1, no. 1, pp. S4-S8. Retrieved from: https://cjasn.asnjournals.org/content/1/Supplement_1/S4. short

[57] Harper, J. L., Conard, M. E., and Besa, E. C. (2020). Iron Deficiency Anemia. Medscape. Retrieved from: https://emedicine.medscape.com/article/202333-overview [accessed April 2, 2019].

[58] Guyatt, G. H., Oxman, A. D., Ali, M., et al. (1992). Laboratory diagnosis of iron deficiency anemia: an overview. Journal of General Internal Medicine, vol. 7, no. 4, pp. 145-153. Retrieved from: https://link.springer.com/article/10. 1007\{\%\}252FBF02598003

[59] Capellini, M. D., Musallam, K. M., and Taher, A. T. (2019). Iron deficiency anemia revisited. Journal of Internal Medicine, vol. 287, no. 2, pp. 153-170. Retrieved from: https://onlinelibrary.wiley.com/doi/full/10.1111/joim.13004

[60] Goddard, A. F., James, M. W., McIntyre, A. S., et al. (2011). Guidelines for the management of iron deficiency anemia. Gut, vol. 60, no. 10, pp. 1309-1316. Retrieved from: https://gut.bmj.com/content/60/10/1309.short

[61] Akarsu, S., Demir, H., Selek, S., et al. (2013). Iron deficiency anemia and levels of oxidative stress induced by treatment modality. Pediatrics International, vol. 55, no. 3, pp. 289-295. Retrieved from: https://onlinelibrary.wiley.com/doi/full/10.1111/ped. 12054 
[62] Casanueva, E. and Viteri, F. E. (2003). Iron and oxidative stress in pregnancy. Gut, vol. 133, no. 5, pp.1700S-1708S. Retrieved from: https://academic.oup.com/jn/article/ $133 / 5 / 1700 S / 4558571$

[63] Auerbach, M. and Shrier, S. (2017). Treatment of iron is getting trendy. The Lancet Haematology, vol. 4, no. 11, p. e500-e501. Retrieved from: https://www.thelancet. com/journals/lanhae/article/PIIS2352-3026(17)30194-1/fulltext

[64] Nair, K. M. (2001). Alternate strategies for improving iron nutrition: lessons from recent research. British Journal of Nutrition, vol. 85, no. 52, pp. S187-S191. Retrieved from: https://www.cambridge.org/core/journals/british-journal-of-nutrition/article/ alternate-strategies-for-improving-iron-nutrition-lessons-from-recent-research/ 9CA74C7D1DDE5D1D5586C87ACDED84C0

[65] Osungbade, K. O. and Oladunjoye, A. O. (2012). Anaemia in developing countries: burden and prospects of prevention and control. In: D. S. Silverberg (Ed.), Anemia. Nigeria: IntechOpen.

[66] Koeller, J. M. and Berg, C. D. (1995). Anemias. In: L. Y. Young and M. A. KodaKimble (Eds.). Applied Therapeutics, The clinical Use of Drugs (6th ed, pp. 881-884). Philadelphia: Lippincott Williams and Wilkins.

[67] Fermandez-Gaxiola, A. C. and De-Regil, L. M. (2019). Intermittent iron supplementation for reducing anemia and its associated impairments in adolescent and adult menstruating women. Cochrane Database of Systematic Reviews. Retrieved from: https://www.cochranelibrary.com/cdsr/doi/10.1002/ 14651858.CD009218.pub3/abstract

[68] Darveau, M., Denault, A. Y., Blais, N., et al. (2004). Bench-to-bedside review: iron metabolism in critically ill patients. Critical Care, vol. 8, pp. 356-362. Retrieved from: https://link.springer.com/article/10.1186/cc2862

[69] Theurl, I., Aigner, A., Theurl, M., et al. (2009). Regulation of iron homeostasis in anemia of chronic disease and iron deficiency anemia: diagnostic and therapeutic implications. Blood, vol. 113, no. 21, pp. 52775285. Retrieved from: https://ashpublications.org/blood/article/113/21/5277/26378/ Regulation-of-iron-homeostasis-in-anemia-of-chronic-renal-failure

[70] Beucher, G., Grossetti, E., Simonet, T., et al. (2011). Iron deficiency anemia and pregnancy. Prevention and treatment. Journal de Gynécologie Obstétrique et Biologie de la Reproduction, vol. 40, no. 3, pp. 185-200. Retrieved from: https: //pubmed.ncbi.nlm.nih.gov/21333465/ 